\title{
Dynamic Failure Characteristics of Metallic Sandwich Plate with Repeated Metallic Truss Cores Subjected to a Plane Strain Drop Impact Condition
}

\author{
Dong-Gyu Ahn ${ }^{1, *}$ and Hyang-Sun Sun ${ }^{2}$
}

\author{
1 Department of Mechanical Engineering, Chosun University, 309 Pilmundaero, Dong-gu, Gwang-ju 501-759, South Korea \\ 2 SEOHAN Inc., 49, 5 Gil, Samsung 1 Ro, Hwaseong-Si, Gyeonggi-do 234-567, South Korea \\ \# Corresponding Author / E-mail: smart@chosun.ac.kr, TEL: +82-62-230-7043, FAX: +82-62-230-7234
}

KEYWORDS : Dynamic failure characteristics, Metallic sandwich plate, Metallic truss cores, Plane strain drop impact, Dynamic failure map, Energy absorption characteristics

\begin{abstract}
Dynamic failure characteristics of a metallic sandwich plate with repeated metallic cores considerably affect the dynamic property and the functionality of the plate. The objective of this paper is to examine dynamic failure characteristics of a metallic sandwich plate with repeated metallic truss cores subjected to plane strain drop impact conditions via a three-dimensional finite element analysis. Dynamic failure modes of the metallic sandwich plate have been estimated using the residual deformed shape of the plate. Nondimensional impact parameters have been adopted to generalize the dynamic failure map (DFM). Limit curves for different dynamic failure modes have been estimated via a regression analysis using the limit impact conditions in dynamic failure plots (DFPs). DFMs of the metallic sandwich plate have been created using the limit curves and the DFPs. The effects of the non-dimensional impact parameters on dynamic failure characteristics of the metallic sandwich plate have been discussed. The relationship between nondimensional impact energy and non-dimensional absorbed energy has been estimated to investigate the energy absorption characteristics of the plate. Finally, it has been revealed that the initiation of the instability mode is prevented to obtain the desired performance of the metallic sandwich plate with repeated metallic truss cores.
\end{abstract}

\section{NOMENCLATURE}

$\varpi=$ mass of impact head

$\mu=$ velocity of impact head

$\mathrm{D}=$ diameter of impact head

$\mathrm{b}=$ width of metallic sandwich plate

$\xi=$ length of specimen

$\rho=$ density of face sheet

$\Phi=$ non-dimensional impact mass

$\mathrm{H}=$ thickness of metallic sandwich plate

$\sigma_{y}=$ reference dynamic yield strength of the face sheet

$\zeta=$ strain rate

$\Pi=$ non-dimensional impact velocity

$\Gamma=$ non-dimensional impact diameter

$\mathrm{S}=$ distance between supports

$\alpha_{i}=$ coefficients of the formula of the limit curves
$\Psi=$ non-dimensional impact energy
$\Omega=$ impact energy
$\mathrm{X}=$ non-dimensional absorbed energy
$\mathrm{E}_{\max }=$ maximum absorbed energy
$\Omega_{\max }=$ maximum impact energy
$\Psi_{\max }=$ maximum non-dimensional impact energy
$\beta_{\mathrm{j}}=$ coefficients of the formula of the N-N curves

\section{Introduction}

One of the recent major issues in the transport vehicle industries is the improvement of energy efficiency through weight reduction and enhancement of structural safety. ${ }^{1-3}$ As an alternative of the conflict demands, a metallic sandwich plate with periodically repeated metallic (PCM) cores has been proposed. ${ }^{4-7}$ Various types of metallic sandwich 
plate with PCM cores have been developed to improve specific stiffness, strength, energy absorption characteristics, and the crash resistance of structural materials and components. ${ }^{8-11}$ In addition, the metallic sandwich plate with PCM cores has potential for multifunctional applications. ${ }^{8-11}$ Dynamic failure characteristics of a metallic sandwich plate with PCM cores considerably affect the dynamic property, the impact resistance and the functionality of the metallic sandwich plate. ${ }^{12-}$ ${ }^{14}$ Hence, the investigation of dynamic failure characteristics is needed to improve the functionality and performance of the metallic sandwich plate with PCM cores under dynamic loading conditions.

Several researchers have investigated the dynamic failure characteristics of metallic sandwich plates with PCM cores to improve the impact resistance and functionality of the metallic sandwich plate. ${ }^{12,13,15-17}$ Lee et al. have investigated the variation of the dynamic failure pattern of a metallic sandwich plate with metallic truss cores according to the strain rate using a Kolsky impact bar and gas gun impact experiments. ${ }^{13}$ Varizi et al. have examined the performance and failure characteristics of metallic sandwich plates with both square honeycomb cores and folded plate cores subjected to intense uniform impulsive pressure loads. ${ }^{15}$ They have estimated the dynamic failure patterns and a dynamic failure map for the metallic sandwich plates. ${ }^{15}$ Yungwirth et al. have created the generalized dynamic failure mechanism map for a metallic sandwich plate with metallic pyramidal truss cores subjected to ballistic impact and clamped conditions. ${ }^{16}$ They have considered tearing and shear-off failures. ${ }^{16}$ Ahn and Jeong have estimated the failure mode and the failure map of a thin metallic sandwich plate with metallic sheared dimple cores under low-velocity impact loading using a finite element analysis. ${ }^{17}$

The objective of this research work is to examine the dynamic failure characteristics of metallic sandwich plate with repeated metallic truss cores subjected to plane strain drop impact conditions via a three-dimensional finite element analysis (FEA). Dynamic failure patterns of the metallic sandwich plate with repeated metallic truss cores for different impact conditions are estimated. Dynamic failure maps (DFMs) of the metallic sandwich plate for different impact conditions are created. In addition, the effects of non-dimensional impact parameters on the dynamic failure mode are investigated using the DFMs. Finally, the relationship between nondimensional impact energy and non-dimensional absorbed energy is estimated to examine the energy absorption characteristics of the metallic sandwich plate with repeated metallic truss cores.

\section{Metallic Sandwich Plate with Repeated Metallic Truss Cores}

Metallic sandwich plate with repeated metallic truss cores are manufactured from multi-points resistance welding of face sheets and metallic truss cores, as shown in Fig $1 .^{18,20}$ Metallic truss cores are
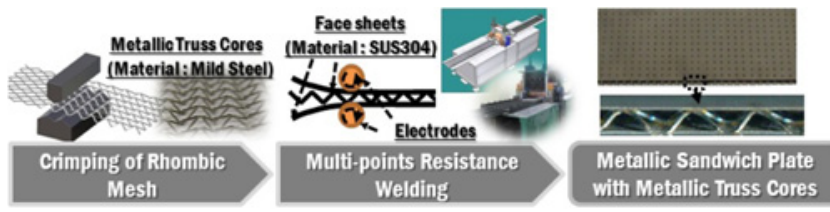

Fig. 1 Manufacturing procedure of the metallic sandwich plate with repeated metallic truss cores fabricated via crimpling of rhombic meshes. ${ }^{18,20}$ Materials of the truss cores and the face sheets are mild steel (CQ grade) and SUS304, respectively. ${ }^{18,20}$ The thicknesses of the metallic sandwich plate and the face sheet are approximately $2.7 \mathrm{~mm}$ and $0.3 \mathrm{~mm}$, respectively. ${ }^{18,20}$ Crimping angle of metallic truss cores is nearly $90^{\circ}$. The relative density of the metallic sandwich plate with repeated metallic truss cores is nearly $0.25 .^{21}$

\section{Finite Element Analysis}

A three-dimensional non-linear FEA is carried out to examine the dynamic failure characteristics of a metallic sandwich plate with repeated metallic truss cores subjected to a plane strain drop impact conditions using ABAQUS V6.11 Explicit. Fig. 2 shows the model of the FE analysis. In order to consider the effects of the initial imperfection of the metallic truss cores on the impact response of the metallic sandwich plate, a symmetric imperfection mode is applied to the metallic truss cores.$^{20} \mathrm{FE}$ meshes are generated for half of the single line of the metallic sandwich plate using the symmetric and the repetition characteristics of the truss cores. ${ }^{17}$ The metallic sandwich plate is represented by 37,200 hexahedron solid element and 52,620 nodes. In order to appropriately simulate the bending behavior of the face sheets and truss cores, the number of layers for the hexahedron elements is chosen to be four. The impact head and fixture are assumed to be rigid bodies. Neo-Hookean strain energy potential is adopted to consider viscoelastic behaviors of the rubber plate at the bottom region of the fixture. ${ }^{17,20}$ True stress-plastic strain relationships with strain rate effects are applied to the FE analysis. Table 1 shows the conditions of FE analysis.

\section{Non-Dimensional Parameters}

Non-dimensional impact parameters are adopted to generalize the DFMs. ${ }^{15-17}$ Eqs. (1), (2) and (3) are the definition of non-dimensional impact mass, non-dimensional impact velocity and non-dimensional impact diameter, respectively. ${ }^{15-17}$ The relationship between the

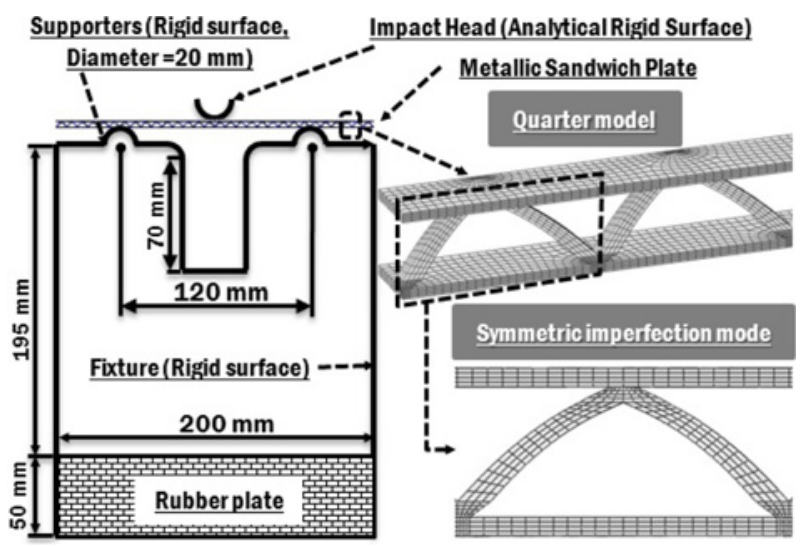

Fig. 2 FE model to simulate the impact response of the metallic sandwich plate subjected to a plane stain drop impact condition

Table 1 Conditions of FE analysis

\begin{tabular}{cccccc}
$\varpi(\mathrm{kg})$ & $\mu(\mathrm{m} / \mathrm{s})$ & $\mathrm{D}\left(\times 10^{-3} \mathrm{~m}\right)$ & $\mathrm{b}(\mathrm{m})$ & $\xi(\mathrm{m})$ & $\rho\left(\mathrm{kg} / \mathrm{m}^{3}\right)$ \\
\hline $0.25-8.33$ & $1.14-2.29$ & $15,30,45$ & 0.06 & 0.2 & 7,850 \\
\hline
\end{tabular}


dynamic yield strength and the strain rate is estimated from high speed tensile tests of the face sheet. Eq. (4) shows the relationship between the dynamic yield strength of the face sheet and the strain rate. The reference dynamic yield strength of the face sheet is calculated from the average of the dynamic yield strength between 10/second and 200/ second of strain rates. ${ }^{17}$

$$
\begin{gathered}
\Phi=\frac{\varpi}{\rho H^{2} b} \\
\Pi=\frac{\mu}{\sqrt{\sigma_{y} \rho}} \\
\Gamma=\frac{D}{S} \\
\sigma_{y}=27.9 \ln (\zeta)+466.1
\end{gathered}
$$

In order to investigate energy absorption characteristics of the metallic sandwich plate, non-dimensional energy parameters, including non-dimensional impact energy and non-dimensional absorbed energy, are adopted. ${ }^{16}$ The non-dimensional impact energy and nondimensional absorbed energy are estimated by Eqs. (5) and (6), respectively. The maximum absorbed energy of the metallic sandwich plate is estimated by direct integration of the contact force versus deflection curve. ${ }^{14}$

$$
\begin{gathered}
\psi=\frac{\varpi \mu^{2}}{2 \sigma_{y} H^{2} b} \\
X=\frac{E_{\max }}{\sigma_{y} H^{2} b}
\end{gathered}
$$

\section{Results and Discussion}

\subsection{Dynamic failure patterns}

The dominant dynamic failure mode of the metallic sandwich plate with repeated metallic truss cores is investigated using the results of the repeated FE analysis for the conditions given in Table 1. The dynamic failure modes of the metallic sandwich plate for different impact conditions are assumed as residual deformed shapes of the metallic sandwich plate after elastic recovery. Through the observation of residual deformed shapes for the metallic sandwich plate, the dominant dynamic failure mode of the metallic sandwich plate with repeated metallic truss cores can be classified into three types, including symmetric buckling mode ( $1^{\text {st }}$ dynamic failure mode), crushing and anti-symmetric buckling mode ( $2^{\text {nd }}$ dynamic failure mode), and instability mode ( $3^{\text {rd }}$ dynamic failure mode), as shown in Fig. 3.

The residual deformed shape of the metallic sandwich plate is similar to its initial shape when the applied impact energy is very small, as shown in Fig. 3(b). The residual deformed shape of the metallic sandwich plate transits from the $1^{\text {st }}$ dynamic failure mode to the $3^{\text {rd }}$ dynamic failure mode when the non-dimensional impact mass and velocity augment, as shown in Figs. 3(c), 3(d) and 3(e). In the $1^{\text {st }}$ dynamic failure mode, symmetric buckling of struts of the metallic truss cores occurs, as shown in Fig. 3(c). In addition, wrinkling and depression of the top face sheet are initiated, as shown in Fig. 3(c).
Through the investigation of the deformation behavior of the metallic sandwich during impact loading and recovery, it is found that rotation of the welded region, which is induced by the difference in buckling deflection between adjacent struts of the metallic truss core, causes wrinkling and depression of the top face sheet, as shown in Fig. 3(c).

Fig. 3(d) shows a residual deformed shape of a metallic sandwich plate with repeated metallic truss cores for the $2^{\text {nd }}$ dynamic failure mode. In the $2^{\text {nd }}$ dynamic failure mode, anti-symmetric buckling and excessive symmetric buckling occur in struts of the $1^{\text {st }}$ line and the $2^{\text {nd }}$ line from the center of the metallic sandwich plate, respectively, unlike the $1^{\text {st }}$ dynamic failure mode. In addition, crushing and wrinkling of the top face sheet appear in the $2^{\text {nd }}$ dynamic failure mode, as shown in Fig. 3(d). This results from the fact that the augmented difference of the buckling deflection between struts of the $1^{\text {st }}$ line and those of the $2^{\text {nd }}$ line increases the rotation angle of the welded region. Contact with the top face sheet and cores is initiated in the $2^{\text {nd }}$ dynamic failure mode, as shown in Fig. 3(d). From the examination of the deformation behavior of the metallic sandwich plate, it is noted that the contact force between the top face sheet and struts of the $2^{\text {nd }}$ line additionally augments the rotation angle of the welded region.

Fig. 3(e) shows the residual deformed shape of the metallic sandwich plate for the $3^{\text {rd }}$ dynamic failure mode. Significant crushing

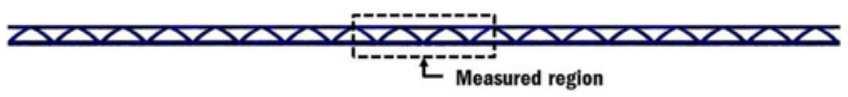

(a) Measured region

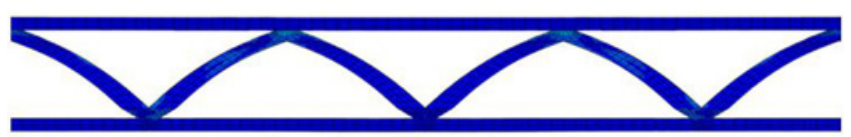

(b) No failure $\left(\Phi=72.9, \Pi=4.17 \times 10^{-3}\right)$

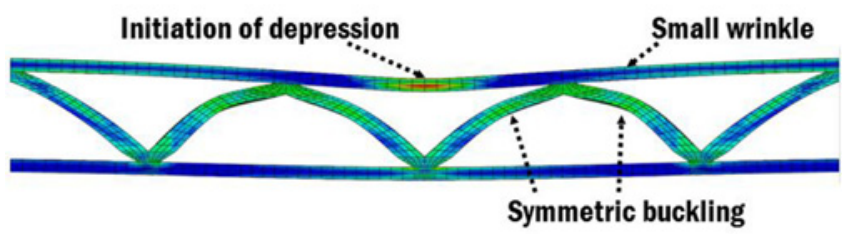

(c) $1^{\text {st }}$ dynamic failure mode $\left(\Phi=145.8, \Pi=5.90 \times 10^{-3}\right)$

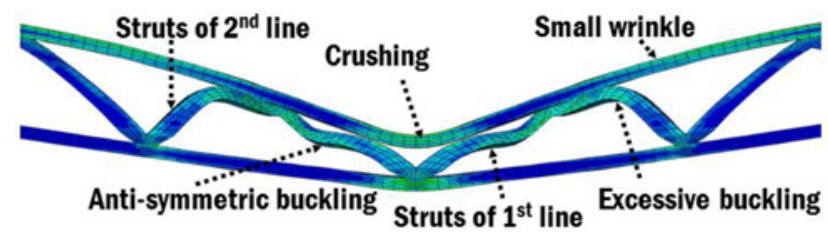

(d) $2^{\text {nd }}$ dynamic failure mode $\left(\Phi=291.7, \Pi=8.35 \times 10^{-3}\right)$

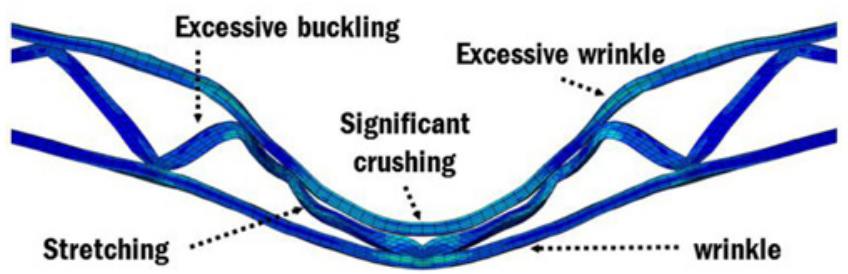

(e) $3^{\text {rd }}$ dynamic failure mode $\left(\Phi=583.4, \Pi=7.23 \times 10^{-3}\right)$

Fig. 3 Dominant dynamic failure mode of the metallic sandwich plate with repeated metallic truss cores $(\mathrm{G}=0.250)$ 
and excessive wrinkling of the top face sheet as well as excessive buckling and stretching of the struts of cores appear in the $3^{\text {rd }}$ dynamic failure mode, as shown in Fig. 3(e). In addition, wrinkling of the bottom face sheet takes place. From the observation of the deformed shape of the metallic sandwich plate during impact loading and recovery, it is noted that excessive wrinkling of the top face sheet and small wrinkles of the bottom face sheet are induced by significant crushing of the struts of the $1^{\text {st }}$ line and the excessive rotation of the welded region, as shown in Fig. 3(e).

\subsection{Dynamic failure maps (DFMs)}

Dynamic failure plots (DFPs) for different non-dimensional impact diameters are created via fitting of each dynamic failure mode for a combination of the non-dimensional impact mass and the nondimensional impact velocity, as shown in Fig. 4. Limit impact conditions for different dynamic failure modes are selected from the DFPs. The limit curve of each dynamic failure mode and the empirical formula of the limit curve are estimated through a regression analysis using the limit impact conditions for different dynamic failure modes, as shown in Fig. 4 and Eq. (7). The exponential function is selected as the basis function of the empirical formula of the limit curves, as shown in Eq. (7). ${ }^{17}$ Table 2 shows the estimated coefficients of the empirical equation for the limit curves.

$$
\Pi\left(\times 10^{-3}\right)=\alpha_{1} \operatorname{Exp}\left(\alpha_{2} \Phi\right)
$$

Using the empirical equations of the limit curves for different dynamic failure modes, the dynamic failure maps (DFMs) for different nondimensional impact diameters are created, as shown in Fig. 4. Fig. 4 shows that extents of no failure, the $1^{\text {st }}$ dynamic failure mode and the $2^{\text {nd }}$ dynamic failure mode augment in the DFMs decrease while the extent of the $3^{\text {rd }}$ dynamic failure mode increases when the non-dimensional impact diameter decreases. Through the investigation of the limit curves for different dynamic failure modes and non-dimensional impact radius, it is observed that the critical non-dimensional impact mass and the critical non-dimensional impact velocity is decreased when the non-dimensional impact diameter is reduced, as shown in Fig. 4. These may be ascribed due to the fact that the increased density of the impact energy, which is induced by the reduction of contact area between the nose of impact head and the specimen, leads to the strain localization of specimen in the vicinity of the impacted region and this, subsequently, causes locally excessive deformation in the vicinity of the impacted region.
The extents of no failure, the $1^{\text {st }}$ dynamic failure mode and the $2^{\text {nd }}$ dynamic failure mode rapidly become narrow when the non-dimensional impact diameter decreases from 0.250 to 0.125 , as shown in Figs. 4 (a) and (b). From this result, it is noted that the strain localization in the vicinity of the impacted region is rapidly accelerated when the nondimensional impact diameter is changed from 0.250 and 0.125 . The limit curve of each failure mode shows a steep slope in the experimental region. This indicates that the effects of the non-dimensional impact velocity on the transition of the dynamic failure mode is larger than the effects of the non-dimensional impact mass on the transition of the dynamic failure mode. Through the comparison of Figs. 4 (a) and (b), it is seen that the influence of the non-dimensional impact velocity on the transition of the dynamic failure mode is conspicuously augmented when the strain localization of the impact region is accelerated.

Using the empirical formula of the limit curve, the maximum nondimensional impact energy is predicted. The maximum nondimensional impact energy is derived from Eq. (5), as shown in Eq. (8). By substituting Eq. (7) for Eq. (8), the formula of the maximum nondimensional impact energy is estimated, as shown in Eq. (9).

$$
\begin{gathered}
\psi_{\max }=\frac{\Omega_{\max }}{\sigma_{y} H^{2} b}=\frac{1}{\sigma_{y} H^{2} b}\left(\frac{\varpi \mu^{2}}{2}\right)_{\max } \\
\psi_{\max }=\frac{\Pi^{2}}{2 \alpha_{1}}\left[\ln (\Pi)-\ln \left(\alpha_{2}\right)\right]
\end{gathered}
$$

\subsection{Energy absorption characteristics}

In order to investigate the energy absorption characteristics of the metallic sandwich plate with repeated metallic truss cores, the relationship between non-dimensional impact energy and non-dimensional absorbed energy is estimated using the results of the FE analysis. Non-dimensional

Table 2 Coefficients of empirical formula of the limit curve

\begin{tabular}{cccc}
\hline$\Gamma$ & Dynamic failure mode & $\alpha_{1}$ & $\alpha_{2}\left(\times 10^{-3}\right)$ \\
\hline \multirow{3}{*}{0.125} & No failure & 8.348 & -4.753 \\
\cline { 2 - 4 } & $1^{\text {st }}$ mode & 9.686 & -2.840 \\
\cline { 2 - 4 } & $2^{\text {nd }}$ mode & 10.996 & -1.271 \\
\hline \multirow{3}{*}{0.250} & No failure & 10.224 & -6.143 \\
\cline { 2 - 4 } & $1^{\text {st }}$ mode & 9.561 & -1.447 \\
\cline { 2 - 4 } & $2^{\text {nd }}$ mode & 11.855 & -1.037 \\
\hline \multirow{3}{*}{0.375} & No failure & 10.224 & -3.767 \\
\cline { 2 - 4 } & $1^{\text {st }}$ mode & 9.692 & -1.162 \\
\cline { 2 - 4 } & $2^{\text {nd }}$ mode & 15.782 & -1.215 \\
\hline
\end{tabular}
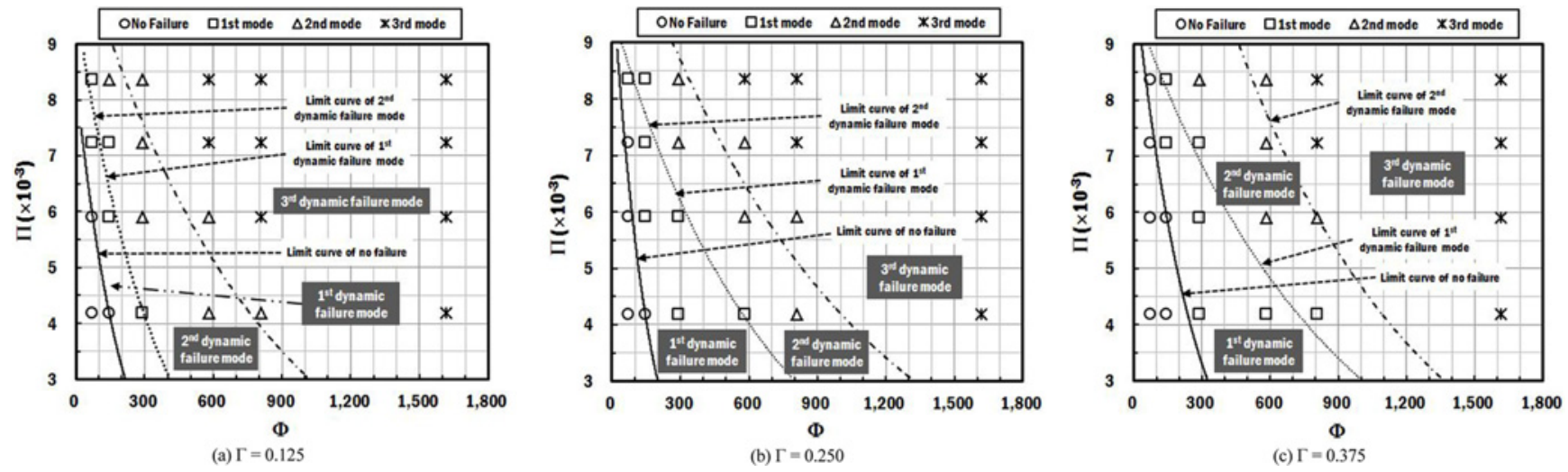

Fig. 4 Dynamic failure plots and dynamic failure maps for different non-dimensional impact diameter 
impact energy versus non-dimensional absorbed energy curves for different non-dimensional impact diameters are predicted by an exponential regression analysis. Eq. (10) is the formula of the nondimensional impact energy - non-dimensional absorbed energy curves. The coefficients of Eq. (10) are calculated by a regression analysis, as shown in Table 3. Using Eq. (10) and Table 3, non-dimensional impact energy versus non-dimensional absorbed energy for the metallic sandwich plate with repeated metallic truss cores is obtained, as shown in Fig. 5.

$$
X=\beta_{1} \Psi^{\beta_{2}}
$$

The critical non-dimensional impact energy, in which the instability mode of the metallic sandwich plate initiates, increases when the nondimensional impact diameter augments, as shown in Fig. 5. The critical non-dimensional impact energy suddenly decreases when the nondimensional impact diameter decreases from 0.250 to 0.125 , as shown in Fig. 5. This result indicates that the applicable impact energy is rapidly reduced when the strain localization is accelerated.

The non-dimensional absorbed energy of the metallic sandwich plate with repeated metallic truss cores is almost identical irrespective of the non-dimensional impact diameter when the non-dimensional impact energy is less than the critical non-dimensional impact energy for the non-dimensional impact diameter of 0.125, as shown in Fig. 5. From this result, it is noted that the performance of the energy absorption of the metallic sandwich plate is hardly influenced by the nondimensional impact energy before initiation of the instability mode. In Fig. 5, it is observed that the non-dimensional absorbed energy of the non-dimensional impact diameter of 0.370 is the same as that of the nondimensional impact diameter of 0.250 . In addition, it is found that the non-dimensional absorbed energy of the non-dimensional impact diameter of 0.125 is smaller than that of the non-dimensional impact diameter of 0.250 when the non-dimensional impact energy is greater

Table 3 Coefficients of formula for non-dimensional impact energy versus non-dimensional absorbed energy curves

\begin{tabular}{ccc}
\hline$\Gamma$ & $\beta_{1}$ & $\beta_{2}$ \\
\hline 0.125 & 1.119 & 1.062 \\
\hline 0.250 & 1.535 & 1.131 \\
\hline 0.375 & 1.470 & 1.118 \\
\hline
\end{tabular}

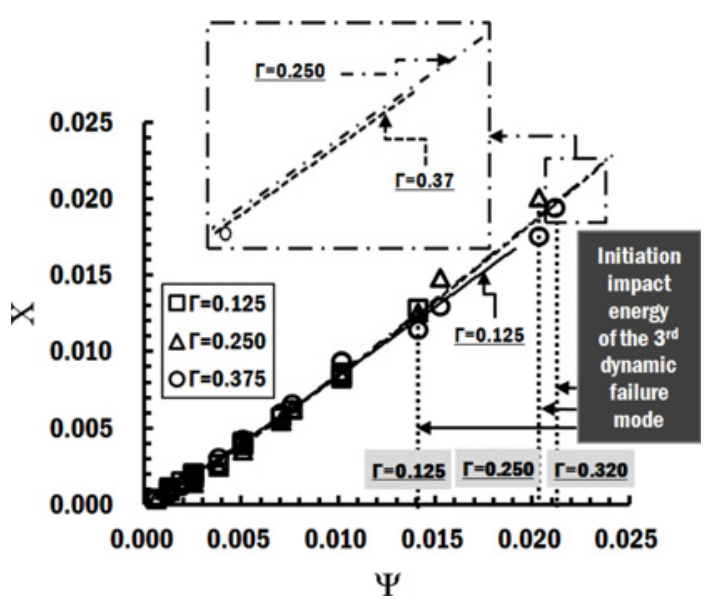

Fig. 5 Non-dimension impact energy versus non-dimensional absorbed energy curves for different non-dimensional impact diameters than the critical non-dimensional impact energy for the non-dimensional impact diameter of 0.125 . These results indicate that the performance of the energy absorption of the metallic sandwich plate conspicuously decreases after initiation of the instability mode of the specimen. From this result, it is considered that the initiation of the instability mode with excessive local deformation of the metallic sandwich plate should be prevented to obtain the desired performance of the metallic sandwich plate with repeated metallic truss cores.

\section{Conclusions}

This paper has investigated the dynamic failure characteristics of a metallic sandwich plate with repeated metallic truss cores subjected to plane strain drop impact conditions via a three-dimensional FE analysis.

Dynamic failure patterns of the metallic sandwich plate with repeated metallic truss cores have been predicted via repeated FE analyses for different impact conditions. Through the investigation of residual deformed shapes of the metallic sandwich for different impact conditions, it has been noted that the dynamic failure mode of the metallic sandwich plate can be classified into symmetric buckling mode ( $\left(1^{\text {st }}\right.$ dynamic failure mode), anti-symmetric buckling mode $\left(2^{\text {nd }}\right.$ dynamic failure mode) and instability mode ( $3^{\text {rd }}$ dynamic failure mode). Non-dimensional impact parameters have been adopted to generalize the DFMs of the metallic sandwich plate with repeated metallic truss cores. The limit curve of each dynamic failure mode and the empirical formula of the limit curve have been estimated via the regression analysis using the limit impact conditions for different dynamic failure modes in the DFPs. DFMs of the metallic sandwich plate have been created using the limit curves of different dynamic failure modes and the DFPs. Using the DFMs, the effects of nondimensional impact parameters on dynamic failure characteristics of the metallic sandwich plate have been discussed. The empirical formula of the maximum non-dimensional impact energy has been predicted using the empirical formula of the limit curve.

In order to investigate the energy absorption characteristics of the metallic sandwich plate with repeated metallic truss cores, the relationship between non-dimensional impact energy and non-dimensional absorbed energy has been estimated. Non-dimensional impact energy versus nondimensional absorbed energy curves for different non-dimensional impact diameters have been predicted by the exponential regression analysis. Through observation of non-dimensional impact energy versus nondimensional absorbed energy curves, it has been noted that the applicable impact energy is rapidly reduced when the strain localization is accelerated. In addition, it has been revealed that the initiation of the instability mode with excessive local deformation is prevented to obtain the desired performance of the metallic sandwich plate.

In the future, the results of this paper should be compared to those of experiments for the improvement of the completeness of this research work.

\section{ACKNOWLEDGEMENT}

This study was supported by research fund from Chosun University, 2013. 


\section{REFERENCES}

1. Kim, J. H., Shin, K. B., and Kim, J. S., "Optimum Design on Suspension Joint Parts of GFRP Composite Bogie Frame with Hshaped Side Beams for Urban Railway Trains,” Int. J. Precis. Eng. Manuf., Vol. 13, No. 1, pp. 71-76, 2012.

2. Jiang, C., Shan, Z., Zhuang, B., Zhang, M, and Xu, Y., "Hot Stamping Die Design for Vehicle Door Beams Using Ultra-high Strength Steel,” Int. J. Precis. Eng. Manuf., Vol. 13, No. 7, pp. 11011106, 2012.

3. Kim, N. and Cha, W. G., "Study on Twisting and Bowing of Roll Formed Products Made of High Strength Steel," Int. J. Precis. Eng. Manuf., Vol. 14, No. 9, pp. 1527-1533, 2013.

4. Choi, J., Shankar K., and Tahtali, M., "Mechanical Behavior and Numerical Analysis of Corrugated Wire Mesh Laminates," J. Mech. Sci. Technol., Vol. 26, No. 1, pp. 73-80, 2012.

5. Choi, S. W., Park, S. H., Jeong, H. S., Cho, J. R., Park S. H., and Ha, M. Y., "Improvement of Formability for Fabricating Thin Continuously Corrugated Structures in Sheet Metal Forming Process,” J. Mech. Sci. Technol., Vol. 26, No. 8, pp. 2397-2403, 2012.

6. Choi, J., Shankar K., Choi, B. B., and Lee, J. H., "In-plane Shear Behavior of Corrugated Cellular Solids and Validation of an Opencell Corrugated Cellular Solid,” Int. J. Precis. Eng. Manuf., Vol. 14, No. 10, pp. 1767-1774, 2013.

7. Nguyen, J., Park, S. I., and Rosen, D., "Heuristic Optimization Method for Cellular Structure Design of Light Weight Components,” Int. J. Precis. Eng. Manuf., Vol. 14, No. 6, pp. 10711078, 2013.

8. Evans, A. G., Hutchinson, J. W., and Ashby, M. F., "Multifunctionality of Cellular Metal Systems," Prog. Mater. Sci., Vol. 43, No. 3, pp. 171-221, 1998.

9. Pingle, S. M., Fleck, N. A., Deshpande, V. S., and Wadley, H. N. G., "Collapse Mechanism Maps for the Hollow Pyramidal Core of a Sandwich Panel under Transverse Shear," Int. J. Solids Struct., Vol. 48, No. 25-26, pp. 3417-3430, 2011.

10. Wadley, H. N. G., "Multifunctional Periodic Cellular Metals," Phil. Trans. R. Soc. A, Vol. 364, No. 1838, pp. 31-68, 2006.

11. Fleck, N. A., Deshpande, V. S., and Ashby, M. F., "Microarchitectured Materials: Past, Present and Future," Proc. R. Soc. A, Vol. 466, No. 2121, pp. 2495-2516, 2010.

12. Xue, Z. and Hutchinson, J., "Crush Dynamics of Square Honeycomb Sandwich Cores,” Int. J. Numer. Mech. Eng., Vol. 65, No. 13, pp. 2221-2245, 2006.

13. Lee, S., Barthelat, F., Hutchinson, J. W., and Espinosa, H. D., "Dynamic Failure of Metallic Pyramidal Truss Core Materials Experiments and Modeling," Int. J. Plasticity, Vol. 22, No. 11, pp. 2118-2145, 2006.
14. Ahn, D. G., Nam, G. H., Seong, D. Y., Han, G. Y., and Yang, D. Y., "Low-Velocity Impact Response of a Thin Metallic Sandwich Plate with a Metallic Sheared Dimple Core Subjected to a Free Boundary Condition,” Mater. Sci. Eng. A, Vol. 532, No. 1, pp. 26-36, 2012.

15. Vaziri, A. Xue, Z., and Hutchinson, J. W., "Performance and Failure of Metal Sandwich Plates Subjected to Shock Loading," J. Mech. Mater. Struct., Vol. 2, No. 10, pp. 1947-1963, 2007.

16. Yungwirth, C. J., Wadley, H. N. G., O’Conner, J. H., Zakraysek, A. J., and Deshpande, V. S., "Impact Response of Sandwich Plates with a Pyramidal Lattice Core,” Int. J. Impact Eng., Vol. 35, No. 8, pp. 920-936, 2008.

17. Ahn, D. G. and Jeong, W. C., "Failure Characteristics of a Thin Metallic Sandwich Plate with Metallic Sheared Dimple Cores under Low-velocity Impact Loading,” J. Mech. Sci. Technol., Vol. 27, No. 10, pp. 2941-2946, 2013.

18. Jung, C. G., Seung, D. Y., Yang, D. Y., Na, S. J., and Ahn, D. G., "Development of a Continuous Fabrication System for a Metallic Sandwich Plate with a Three-dimensional Truss Core," Int. J. Adv. Manuf. Technol., Vol. 45, No. 3-4, pp. 352-361, 2009.

19. Seong, D. Y., Jung, C. G., Yang, D. Y., and Chung, W. J., "Efficient Prediction of Local Failures for Metallic Sandwich Plate with Pyramidal Truss Cores during the Bending Process,” Int. J. Precis. Eng. Manuf., Vol. 12, No. 3, pp. 491-503, 2011.

20. Ahn, D. G. and Sun, H. S., "Imperfection Mode of Thin Metallic Sandwich Plate with Pyramidal Metallic Inner Structures," Trans. Korean Soc. Mech. Eng. A., Vol. 37, No. 2, pp. 187-192, 2013.

21. Ahn, D. G., Lee, S. H., Kim, M. S., Han, G. Y., Jung, C. G, and Yang, D. Y., "Investigation into Characteristics of Bending Stiffness and Failure for ISB Panel," J. Korean Soc. Precis. Eng., Vol. 22, No. 9, pp. 162-172, 2005. 